\title{
The Synchronization of Two Metriplectic Systems Arisen from the Lü System
}

\author{
Camelia Pop Ariesanu \\ "Politehnica" University of Timisoara, Math Department, 300002, 2, Victoriei PI., Timisoara, \\ Romania \\ camelia.ariesanu@mat.upt.ro, cariesanu@yahoo.com
}

Keywords: Hamilton-Poisson realization, metriplectic system, synchronization, asymptotic stability.

Abstract. The concept of the metriplectic system was introduced in 1980's by Kaufman (see [1]). These have important applications in a lot of different mathematical fields, in fluid mechanics or information security. Beginning with the Hamilton-Poisson realization of the Lü system (see [2] for details) we construct the associated metriplectic system by adding a dissipation term of a special form. Then, using Pecora and Carroll method, we discuss the synchronization of the two coupled metriplectic systems. In the last part we present an example where the synchronization is used.

\section{Introduction}

The original Lü system (see [3] for details) of differential equations on $R^{3}$ has the following form:

$$
\left\{\begin{array}{c}
\dot{x}_{1}=\alpha\left(x_{2}-x_{1}\right) \\
\dot{x}_{2}=-x_{1} x_{3}+\beta x_{2} \\
\dot{x}_{3}=x_{1} x_{2}-\gamma x_{3}
\end{array}\right.
$$

where $\alpha, \beta, \gamma \in R$.

The main purpose of the paper is to analyze the synchronization of two metriplectic systems associated to the Hamilton-Poisson realization of the Lü system and to show how we can use this to secure the communication. First of all, let us make a short resume of the definitions of the Hamilton-Poisson geometry and the metriplectic systems.

Definition $1([4,5])$ If $M$ is a smooth manifold and $C^{\infty}(M)$ is the set of the smooth real functions on $M$, then a Poisson bracket on $M$ is a bilinear and skew-symmetric map:

$(F, G) \rightarrow\{F, G\} \in C^{\infty}(M), \quad F, G \in C^{\infty}(M)$

such that the Jacobi identity and the Leibniz rule are verified, i.e. the following properties hold:

$-\{F, G\}=-\{G, F\}$;

$-\{F,\{G, H\}\}+\{G,\{H, F\}\}+\{H,\{F, G\}\}=0$;

$-\{F, G \cdot H\}=\{F, G\} \cdot H+G\{F, H\}$.

Proposition 1. ([4,5]) If $\{\cdot, \cdot\}$ is a Poisson structure on $R^{3}$ and $F, G \in C^{\infty}\left(R^{3}, R\right)$ then we have:

$\{F, G\}=\sum_{i, j=1}^{3}\left\{x_{i}, x_{j}\right\} \frac{\partial F}{\partial x_{i}} \frac{\partial G}{\partial x_{j}}$.

Let us take the matrix $\Pi$ given by $\Pi=\left[\left\{x_{i}, x_{j}\right\}\right]$.

Proposition 2. $([4,5])$ Any Poisson structure $\{\cdot, \cdot\}$ on $R^{3}$ is uniquely determined by the matrix $\Pi$ using the formula:

$\{F, G\}=(\nabla F)^{t} \Pi(\nabla G)$.

Definition 2. ([4,5]) The triple $\left(R^{3},\{\cdot, \cdot\}, H\right)$ where $\{\cdot, \cdot\}$ is a Poisson bracket on $R^{3}$ and $H \in C^{\infty}\left(R^{3}, R\right)$ is the Hamiltonian is a Hamilton-Poisson system on $R^{3}$.

The dynamics of a Hamilton-Poisson system can be written in the following form:

$$
\dot{x}=\Pi \cdot \nabla \mathrm{H}, \quad x=\left(x_{1}, x_{2}, x_{3}\right)^{t} .
$$


Definition 3. ([4, 5]) If $\{\cdot, \cdot\}$ is a Poisson structure on $R^{3}$ then a function $C \in C^{\infty}\left(R^{3}, R\right)$ which satisfies:

$\{F, C\}=0, \quad \forall F \in C^{\infty}\left(R^{3}, R\right)$.

is a Casimir of the Poisson structure.

Now, we add to the Hamilton-Poisson system (2) a dissipation term of the form $M \cdot \nabla \tilde{C}$, where $M$ is a symmetric matrix which satisfies certain compatibility conditions and $\tilde{C}=a C, a \in R$ and $C \in C^{\infty}\left(R^{3}, R\right)$ is a Casimir function. The systems of the form:

$$
\dot{x}=\Pi \cdot \nabla \mathrm{H}+M \cdot \nabla \tilde{C}
$$

are a family of metriplectic systems with the same Hamiltonian $H$.

In fact, the metriplectic systems (3) can be viewed as a perturbation of the Hamilton-Poisson system (2), for each $a \in R$. hold:

Definition 4. ([6, 7]) The system (3) is a metriplectic system in $R^{3}$ if the following conditions

i. $\quad M \cdot \nabla H=0$;

ii. $\quad(\nabla \tilde{C})^{t} \cdot M \cdot \nabla \tilde{C} \leq 0$.

The system (3) is the metriplectic system associated to the Hamilton-Poisson system (2).

For more details concerning the Hamilton-Poisson systems and metriplectic systems see [4, 5, 6, 7].

The paper is structured as follows: the second paragraph presents a case for which the Lü system admits a Hamilton-Poisson realization and the metriplectic system associated to this realization. Some properties regarding the stability problems are presented here, too. The synchronization problem for dynamical systems has received a great deal of interest due to its application in a lot of different fields of science, so the third part of the paper is dedicated to this subject. Numerical simulations obtained via MATHEMATICA 7.0 are presented, too. In the last section we gathered the facts presented along this paper and we applied them in secure communication.

\section{The Metriplectic System Associated to the Lü System}

Let us begin this section by presenting the Hamilton-Poisson structure of the Lü system.

Proposition 3 ([2]) If $\alpha=\beta=\gamma=0$, the Lü system has the Hamilton-Poisson realization $\left(R^{3}, \Pi, H\right)$ where:

$$
\Pi=\left(\begin{array}{ccc}
0 & x_{1} x_{3} & -x_{1} x_{2} \\
-x_{1} x_{3} & 0 & -x_{1} \\
x_{1} x_{2} & x_{1} & 0
\end{array}\right)
$$

and

$$
H\left(x_{1}, x_{2}, x_{3}\right)=\frac{1}{2}\left(x_{2}^{2}+x_{3}^{2}\right) .
$$

Remark: ([2]) The configuration described in the Proposition 3 has one functionally independent Casimir, given by the function

$$
C: R^{3} \rightarrow R, C\left(x_{1}, x_{2}, x_{3}\right)=2 x_{1}-x_{2}^{2}-x_{3}^{2} .
$$

Starting with the Hamilton-Poisson realization of the Lü system presented in the Proposition 3, we shall focus now on constructing its associated metriplectic system. In order to do this we determine the symmetric matrix $\boldsymbol{M}=\left(\boldsymbol{m}^{i j}\right)$, where:

$$
m^{i i}(x)=-\sum_{k=1, k \neq i}^{3}\left(\frac{\partial H}{\partial x_{k}}\right)^{2} \text { and } m^{i j}(x)=\frac{\partial H}{\partial x_{i}} \frac{\partial H}{\partial x_{j}} \text {, for } i \neq j .
$$


By applying (7) to the Hamiltonian function (5) we obtain the symmetric matrix Mgiven by:

$$
M=\left(\begin{array}{ccc}
-x_{2}^{2}-x_{3}^{2} & 0 & 0 \\
0 & -x_{3}^{2} & x_{2} x_{3} \\
0 & x_{2} x_{3} & -x_{2}^{2}
\end{array}\right)
$$

For the function $\tilde{C}=a C, a \in R$, where $C$ is the Casimir function (8) of the Poisson configuration $\Pi$, the differential system in the tensorial form:

$$
\dot{x}_{i}=\Pi^{\mathrm{ij}} \frac{\partial H}{\partial x_{j}}+m^{i j} \frac{\partial \tilde{C}}{\partial x_{j}}, i, j=\overline{1,3 .}
$$

is the metriplectic system associated to a Hamilton-Poisson system. Using (5), (6) and (8) the system (9) becomes:

$$
\left\{\begin{array}{c}
\dot{x}_{1}=-2 a\left(x_{2}^{2}+x_{3}^{2}\right) \\
\dot{x}_{2}=-x_{1} x_{3} \\
\dot{x}_{3}=x_{1} x_{2}
\end{array}, \quad a \in R .\right.
$$
on $R^{3}$.

Proposition 2. The dynamical system $\left(R^{3}, \Pi, H, M, \tilde{C}\right)$ given by (10) is a metriplectic system Proof: The function $C$ is a Casimir of the Hamilton-Poisson system $\left(R^{3}, \Pi, H\right)$. We have

$M \cdot \nabla H=0$ and:

$$
(\nabla \tilde{C})^{t} \cdot M \cdot \nabla \tilde{C}=-4 a^{2}\left(y^{2}+z^{2}\right) \leq 0 .
$$

Hence (10) is a metriplectic system.

Proposition 3. (i) The function $H$ given by (5) is a constant of motion for the metriplectic system (10).

(ii) The function $\tilde{C}$ decreases along the solution of the system (10).

Proof: (i) We have $d H=0$.

(ii) The derivative of $\tilde{C}$ along the solutions of the system (10) verifies the condition

$$
\frac{d \tilde{C}}{d t} \leq 0
$$

We will study now some dynamical properties of the system (10). If $a \in R^{*}$ its equilibrium states are given by the family:

$$
e^{M}=(M, 0,0), M \in R \text {. }
$$

The results about the stability of the states $e^{M}$ are presented in the following:

Proposition 4. If $a \in R^{*}$ the equilibrium states $e^{M}$ are spectrally stable for any $M \in R$.

Proof: The characteristic polynomial of the matrix corresponding to the linear part of the system (10) at the equilibrium of interest has the following roots:

$\lambda_{1}=0, \lambda_{2,3}= \pm i M$

and then our assertion immediately follows.

Now, if $a=0$, the equilibrium states of the system (10) are

$$
e^{M}=(M, 0,0), \quad e^{N, P}=(0, N, P), M, N, P \in R \text {. }
$$

Proposition 5 ([2]) If $a=0$ the equilibrium states $e^{M}$ are spectrally stable for any $M \in R$. The stability of the equilibrium states $e^{N, P}$ remains an open problem. 


\section{The synchronization of two metriplectic systems}

For constructing the drive-response configuration, we will use Pecora and Caroll method (see [8]).

The metriplectic system (10) will be the drive system; this system and the response system are the configuration and we suppose that these systems are coupled. This means that the response system is driven by the drive one such that the behavior of each of them is not affected by the other. So, the drive system is:

$$
\left\{\begin{array}{c}
\dot{x}_{1}=-2 a\left(x_{2}^{2}+x_{3}^{2}\right) \\
\dot{x}_{2}=-x_{1} x_{3} \\
\dot{x}_{3}=x_{1} x_{2}
\end{array}\right.
$$

and the response system:

$$
\left\{\begin{array}{c}
\dot{y}_{1}=-2 a\left(y_{2}^{2}+y_{3}^{2}\right)+u_{1} \\
\dot{y}_{2}=-y_{1} y_{3}+u_{2} \\
\dot{y}_{3}=y_{1} y_{2}+u_{3}
\end{array}\right.
$$

where $u_{1}(t), u_{2}(t), u_{3}(t)$ are three control functions.

The synchronization error system is the difference between the metriplectic system (12) and the controlled system (11):

$$
e_{i}(t)=y_{i}(t)-x_{i}(t), \quad i=1,2,3 \text {. }
$$

Using (11) and (12) one can get:

$$
\left\{\begin{array}{c}
\dot{e}_{1}=-2 a\left(2 x_{2} e_{2}+2 x_{3} e_{3}+e_{2}^{2}+e_{3}^{2}\right)+u_{1} \\
\dot{e}_{2}=-x_{1} e_{3}-x_{3} e_{1}-e_{1} e_{3}+u_{2} \\
\dot{e}_{3}=x_{1} e_{2}+x_{2} e_{1}+e_{1} e_{2}+u_{3}
\end{array}\right.
$$

A good way to define the controls $u_{1}, u_{2}, u_{3}$ is the following:

$$
\left(\begin{array}{l}
u_{1} \\
u_{2} \\
u_{3}
\end{array}\right)=A\left(\begin{array}{l}
e_{1} \\
e_{2} \\
e_{3}
\end{array}\right)+\left(\begin{array}{c}
2 a\left(e_{2}^{2}+e_{3}^{2}\right) \\
e_{1} e_{3} \\
-e_{1} e_{2}
\end{array}\right)
$$

where

$$
A=\left(\begin{array}{ccc}
-1 & 0 & 0 \\
x_{3} & -1 & 0 \\
-x_{2} & -x_{1} & -1
\end{array}\right)
$$

i.e.

$$
\left\{\begin{array}{c}
u_{1}=-e_{1}+2 a\left(e_{2}^{2}+e_{3}^{2}\right) \\
u_{2}=x_{3} e_{1}-e_{2}+e_{1} e_{3} \\
u_{3}=-x_{2} e_{1}-x_{1} e_{2}-e_{3}-e_{1} e_{2}
\end{array}\right.
$$

Using (14) the system's error becomes:

$$
\left\{\begin{array}{c}
\dot{e}_{1}=-e_{1}-2 a\left(2 x_{2} e_{2}+2 x_{3} e_{3}\right) \\
\dot{e}_{2}=-e_{2}-x_{1} e_{3} \\
\dot{e}_{3}=-e_{3}
\end{array}\right.
$$

Proposition 6 The equilibrium state $(0,0,0)$ of the system $(15)$ is asymptotically stable. Proof: It is easy to see that all the conditions of the Lyapunov-Malkin Theorem (see [9] for details) are satisfied, so the equilibrium state $(0,0,0)$ of the system $(15)$ is asymptotically stable. 
Remark: Numerical simulations using fourth-order Runge-Kutta integrator are presented in the Figure 1 for the system (15) with the controls $u_{i}, i=1,2,3$ given by (14); the initial states of the drive system and the response system are $x_{1}(0)=x_{2}(0)=x_{3}(0)=0.1$ respectively $y_{1}(0)=$ $y_{2}(0)=y_{3}(0)=0.1, e_{1}(0)=0, e_{2}(0)=e_{3}(0)=0$ and $a=1$. One can see that each error converges to 0 .
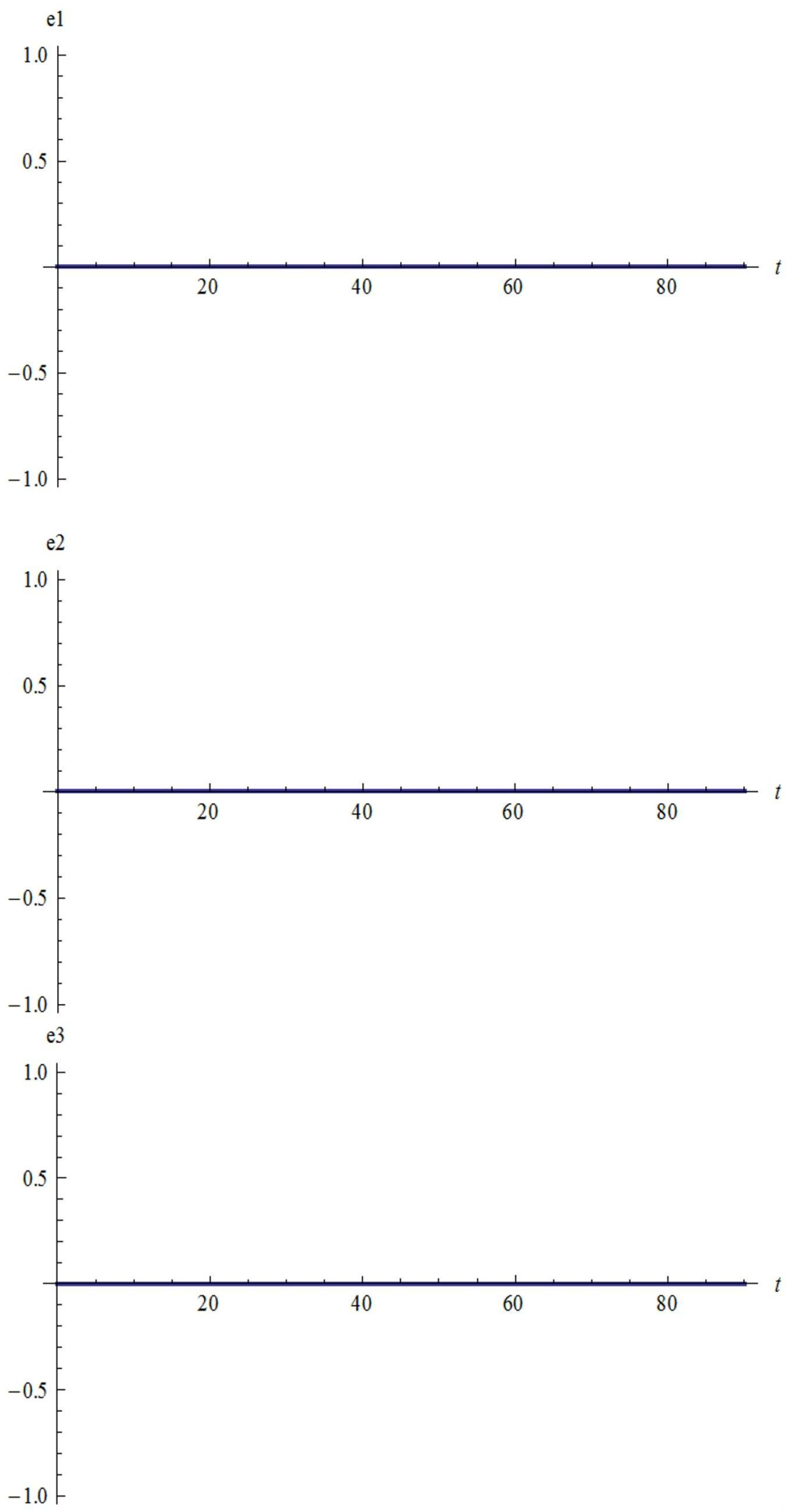

Figure 1 The solutions $e_{1}, e_{2}, e_{3}$ of the system (15) 
Remark: The following figures present the synchronization of the systems (11) and (12) with the controls given by (14) for $a=1$.
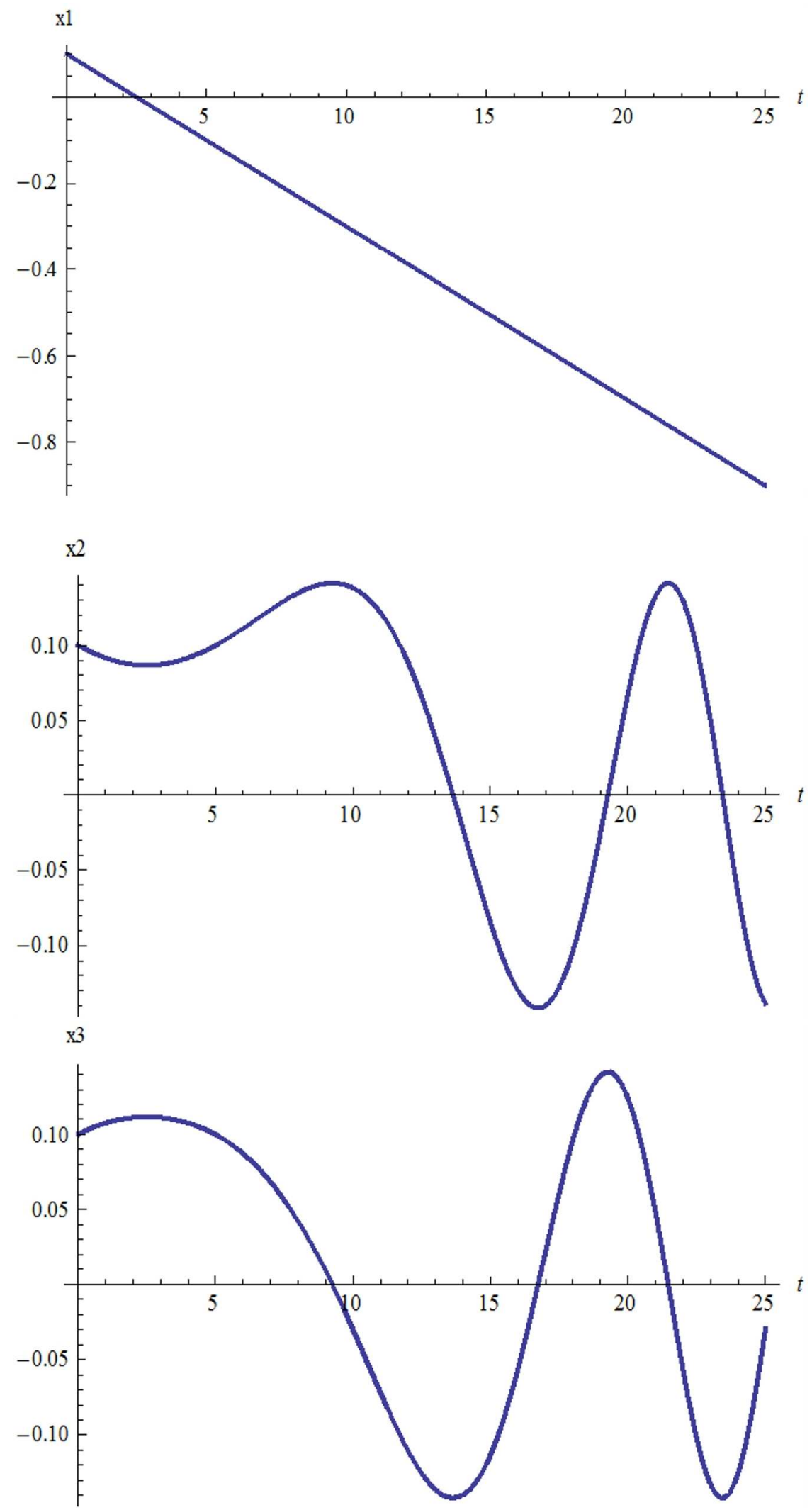

Figure 2 The solutions $x_{1}, x_{2}, x_{3}$ of the system (11) 

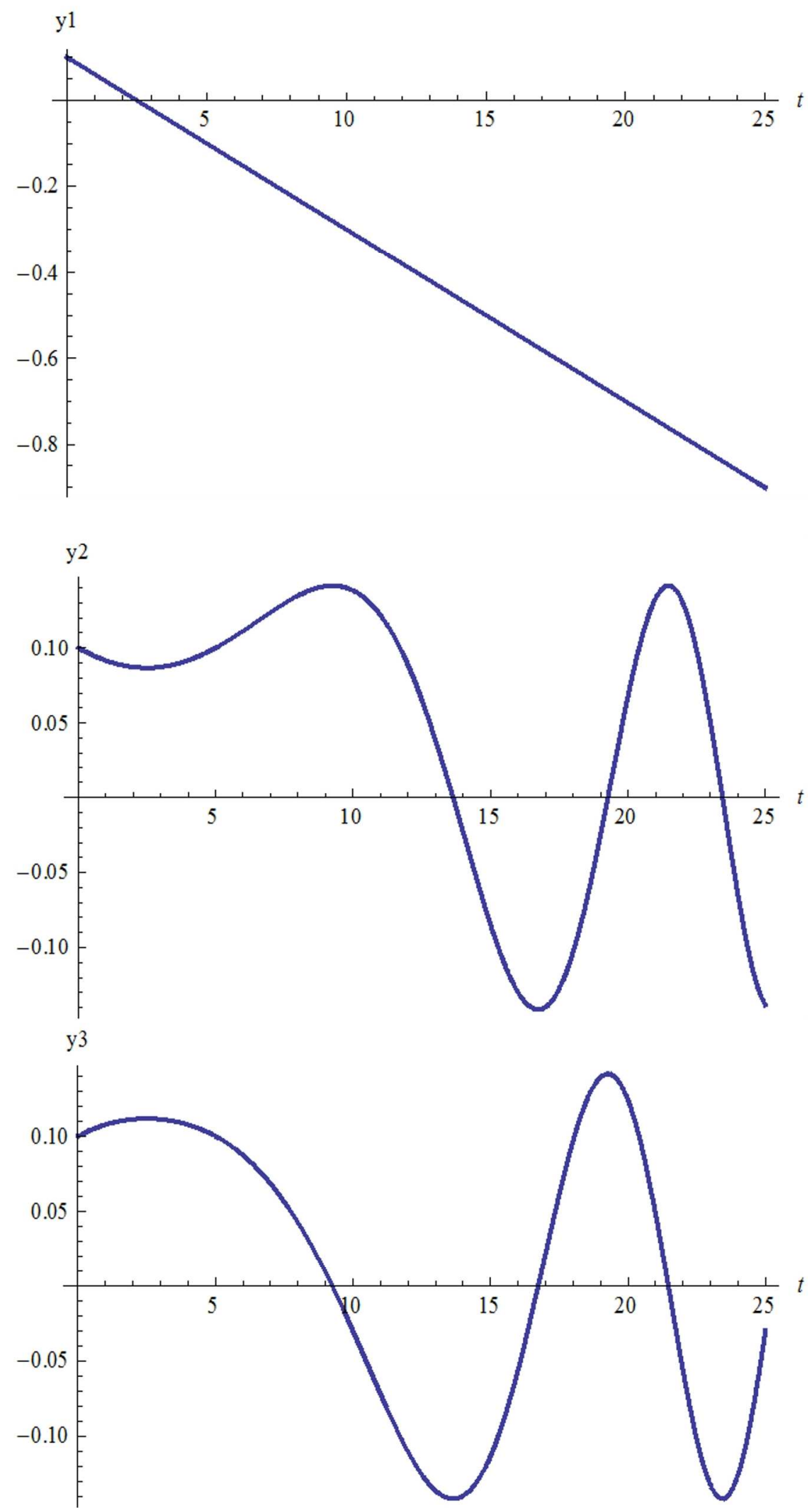

Figure 3 The solutions $y_{1}, y_{2}, y_{3}$ of the system (12) with the controls (14)

\section{Cryptography using the synchronized systems (11) and (12)}

In this section we will use the synchronized systems (11) and (12) to the communication using the cryptographic encode (see [10] for details). We can see that the errors $e_{1}, e_{2}, e_{3}$ converges to zero for any $t>0$ so we have $x_{i}(t)=y_{i}(t)$ for any $t>0$. The message that a sender wants to send to the receiver is called plaintext (p) and its correspondent by decryption is called ciphertext (c). The 
plaintext and the cipher text are represented by numbers, each letter being replaced by a corresponding number, so instead of 26 letters we will use the numbers between 0 and 25 . The sender uses the dynamical system (11) and the receive uses (12). They choose values for variables $x_{1}(t)$ and $y_{1}(t)$ as secret keys after a period $t=2$, for instance, when the synchronization between the two systems is achieved. The corresponding formula for the ciphertext message is

$c=p+k \bmod 26$

and decrypted message can be obtained by:

$p=c-k \bmod 26$

where $k$ is the secret key. Corresponding to every message letter we use one and only one message key which are randomly generated. In fact, the key $k$ hide and secure the plaintext $p$.

Let us consider the message ,TIMISOARA“. We assign the numbers $0 \ldots 25$ to the letters A...Z. To take the values of the secret keys as integer, we choose $100 \cdot x_{1}(t)$ and $100 \cdot y_{1}(t)$ respectively. The data from $x_{1}(t)$ picked up by the sender and the corresponding key are presented in Table1.

Table 1 The cyphertext sent

\begin{tabular}{|c|c|c|c|c|}
\hline$t$ & $x_{1}(t)$ & $k$ & $p$ & $c=p+k \bmod 26$ \\
\hline 2 & 0.02 & 2 & T 19 & 21 \\
\hline 3 & -0.02 & -2 & I 8 & 6 \\
\hline 4 & -0.06 & -6 & M 12 & 6 \\
\hline 5 & -0.1 & -10 & I 8 & 24 \\
\hline 6 & -0.14 & -14 & S 18 & 22 \\
\hline 7 & -0.18 & -18 & O 14 & 0 \\
\hline 9 & -0.26 & -26 & A 0 & 13 \\
\hline 10 & -0.3 & -30 & R 17 & 8 \\
\hline 11 & -0.34 & -34 & A 0 & \\
\hline
\end{tabular}

For every letter a key is generated. The message arrives encrypted to the second person together with the key. The second person uses the component $y_{1}(t)$ to decrypt the message.

If the message does not contain only letters, we can generalize the method presented above: for every small letter, upper letter or special character we associate a number from in ASCII representation.

Table 2 The plaintext recovered

\begin{tabular}{|c|c|c|c|c|}
\hline$t$ & $y_{1}(t)$ & $k$ & $c$ & $p=c-k \bmod 26$ \\
\hline 2 & 0.02 & 2 & 21 & $19(\mathrm{~T})$ \\
\hline 3 & -0.02 & -2 & 6 & $8(\mathrm{I})$ \\
\hline 4 & -0.06 & -6 & 6 & $12(\mathrm{M})$ \\
\hline 5 & -0.1 & -10 & 24 & $8(\mathrm{I})$ \\
\hline 6 & -0.14 & -14 & 4 & $18(\mathrm{~S})$ \\
\hline 7 & -0.18 & -18 & 22 & $0(\mathrm{O})$ \\
\hline 9 & -0.26 & -26 & 0 & $17(\mathrm{R})$ \\
\hline 10 & -0.3 & -30 & 13 & $0(\mathrm{~A})$ \\
\hline 11 & -0.34 & -34 & 8 & \\
\hline
\end{tabular}

\section{Conclusion}

The paper presents a chaotic system obtained from a special case of the Lü system. By adding a dissipation term to a Hamilton-Poisson system we obtain a metriplectic system on $R^{3}$. This method is presented in [7]. In the last part, the synchronization problem of the two metriplectic systems is presented. Since a suitable control has been chosen we get the synchronization of the two systems. Using the software MATHEMATICA 7.0. the numerical simulations are obtained for the specific 
case $a=1$. To solve the systems (11), (12) and (15) with the control functions $u_{1}, u_{2}, u_{3}$ given by (14) we use the fourth-order Runge-Kutta integrator. The synchronized systems are used to encrypt and decrypt a message. This is only one application of the metriplectic systems. Other applications are presented in [11].

\section{Acknowledgment}

This paper was supported by the project "Development and support of multidisciplinary postdoctoral programs in major technical areas of national strategy for Research - Development Innovation" 4D-POSTDOC, contract no. POSDRU/89/1.5/S/52603, project co-funded by the European Social Fund through Sectorial Operational Program Human Resources Development 2007-2013.

\section{References}

[1] A. N. Kaufman, Dissipative Hamiltonian systems: A unifying principle, Phys. Lett. A 100, 8 , (1984), 419 - 422; MR0735549 (85j:58058).

[2] C. Pop (Ariesanu), C. Petrisor, D. Bala, Hamilton-Poisson Realizations for the Lü System, Mathematical Problems in Engineering, Vol. 2011, Article ID 842325, doi:10.1155/2011, MR2781571 (2012a:37040)

[3] J. Lü, G. Chen, A new chaotic attractor coined, Internat. J. Bifur. Chaos Appl. Sci. Engrg, 12(3) (2002) 659-661.

[4] J. M. Ginoux, B. Rossetto, Differential geometry and mechanics: Applications to chaotic dynamical systems, Int. J. Bifurcation Chaos Appl. Sci. Engrg., 16(4), (2006), 887-910.

[5] M. Hirsch, S. Smale, R. Devaney, Differential Equations, Dynamical Systems and an Introduction to Chaos, Elsevier, Academic Press, New York, 2003.

[6] P. Guha, Metriplectic structure, Leibniz dynamics and dissipative systems, J. Math. Anal. Appl., 326 (2007), Issue 1, 121 - 136.

[7] Gh. Ivan, D. Opris, Dynamical systems on Leibniz algebroids, Differ. Geom. Dyn. Syst., 8(2006), 127 - 137 (electronic). MR2220718 (2006m:53130).

[8] L.M. Pecora and T.L. Carroll: Synchronization in chaotic systems, Phys. Rev. Lett., 64(8) (1990), $821-824$.

[9] D. Zenkov, A. Bloch and J. Marsden: The energy-momentum method for the stability of nonholonomic systems, Dynam. Stability Systems, 13(1998), no.2, 123 - 156.

[10] D. Ghosh, S. Banerjee, A. R. Chowdhury, Synchronization between variable time-delayed systems and cryptography. Europhys. Lett. EPL 80 (2007), no. 3, Art. 30006, 6 pp. MR2354131 (2008j:94035).

[11] P. Birtea, M. Boleantu, M. Puta, R. M. Tudoran, Asymptotic stability for a class of metriplectic systems, J. Math. Phys. 48, no. 8 (2007). 